\title{
SINDROME DE COMPRESSÃO DA VEIA CAVA INFERIOR NA PARACOCCIDIOIDOMICOSE
}

Silvino alves de CARvabho (1), Giovanni Guido CERRI (2), Mario Shiroma (3), Maria Aparecids SHLKANAT-YaSUDa (4), Antonio Alci BARONE (5) e Vicente AMATO NETO (6)

\section{R E S M O}

Os Autores registram o primeiro caso de síndrome de compressão da veia cava inferior devida a paracoccidioidomicose. Resumem a evolução clínica do paciente, tratamento e os achados laboratoriais e ultrassonográficos que evidenciaram o diagnóstico de compressăo da veia cava inferior.

UNITERMOS: Paracoccidioidomicose humana - Compressão da veia cava inferior - Ultrassonografia.

\section{NTRODU G $\mathbf{A} O$}

A paracoccidioidomicose representa em alguns paises da América Latina, e em especial no Brasil, grave problema das doenças infectoparasitárias.

Causada pelo fungo P. brasiliensis (SPLENDORE, 1912) ALMEIDA, 1930 7, a paracoccidioidomicose se constitui numa infecção de caráter sistêmico, acometendo principalmente os órgãos respiratórios, o sistema linfo-glandular e o tegumento. Entretanto, pelas suas características fisiopatogênicas e seu caráter invasivo, qualquer órgăo ou sistema pode ser acometido pelo $\mathbf{P}$. brasiliensis.

Dados da literatura revelam acometimento dos mais variados órgãos: sistema nervoso central ${ }^{8}$, fígado e vias biliares 2,16 , pâncreas ${ }^{12}$, aparelho digestivo (esôfago, estômago e intestinos) 1.540 . peritôneo 5 , glândulas supra-renais ${ }^{7}$, ossos 3, miocárdio 13, enđocárợio. testículos 9 e vias respiratórias 6,15 .

O acometimento do sistema linfo-glandular pode levar a mecanismos compressivos de orgãos intra-abdominais. Entretanto não há relato na literatura de compressão de veia cava na paracoccidioidomicose e que seria o objeto desse relato.

\section{REGISTRO DO CASO}

Paciente de 41 anos, amarelo, masculino, apresentou em 1972 quadro de semi-oclusão intestinal: Submetido à laparotomia exploradora comprovou-se histologicamente paracoccidioidomicose. Com anfotericina $B$ teve excelenie resposta terapêutica. Fez uso de sulfadoxina de manutenção até 1975, quando perdeu-se o se-

(1) Professor-assistente da Clinica de Doenças Infecciosas e Parasitárias da Faculdade de Medicina da Universidade de Sâo Pauro

(2) Professor-assistente doutor do Departamento de Radiologia da Faculdade de Medicina da Universidade de Săo Paulo

(3) Professor-adjunto da Clínica de Doenças Infecciosas e Parasitárias da Faculdade de Medicina a Universidade de słáo Paulo

(4) Professora-assistente doutora da Clinica de Doenças Infecciosas e Parasitárias da Faculdade de Medicina ta Uni. versidado de Sāo Paulo

(5) Médico-assistente da Clínica de Doenças Infecciosas e Parasitárias do Hospital das CIfnicas da Faculdade de Medi cina da Universidade dẹ São Paulo

(6) Professor-titutar da Clinica de Doencas Infeccioses e Parasitarias da Faculdade de Medicina da Universidade de Săo Paulo 
CARVALHO, S. A. de: CERRI. G. G.; SHIROMA, M,; SHLKANAIYASUDA. M. A.; BARONE, A. A. \& AMATO NETO, V. - Sindrome de compressîo da veia cava inferior na paracoccidioidomicose. Rev. Inst. Med. trop. Sä́o Paulo zs: $61-64,1986$

guimento. Em 22-04-82 retornou com acometimento ganglionar periférico e lesčes de oro fa ringe. Reinternado, constatou-se: presença de P. brasiliensis em material de oro-faringe, contraimunoeletroforese $1 / 32$ e a ultrassonografia abdominal realizada em 27-05-82 (Fig. 1), evidenciou adenomegalia no hilo hepático e cadeia para-aórtica. Em 28-05-82 medicado com ketoconazol ( $400 \mathrm{mg} /$ dia) regularmente por dois meses e desde então usou irregularmente o medicamento. Em 07-10-82 retornou com aumento do volume abdominal e edema dos membros inferiores. Constatou-se, ao exame clinico, ede- ma $(+t+)$ e ausência de ascite ou massas palpaveis no abdomem. Em 14-1082 a ultrassonografia de abdomem (Fig. 2) revelou a veia cava inferior reduzida de calibre, com imagens hipoecogênicas múltiplas (PRE-CAVA) em topografia peri-cava caracterizando uma linfoadenopatia peri-cava com compressão da mesma. Foi reintroduzida a terapêtica com ketoconazol (400 mg/dia). Em 24-11-82 foi feita linfografia, cujo resultado foi normal e em 13-12-82 foi realizada nova ultrassonografia (Fig. 3) que năo evidenciou linfoadenopatia peri-cava.

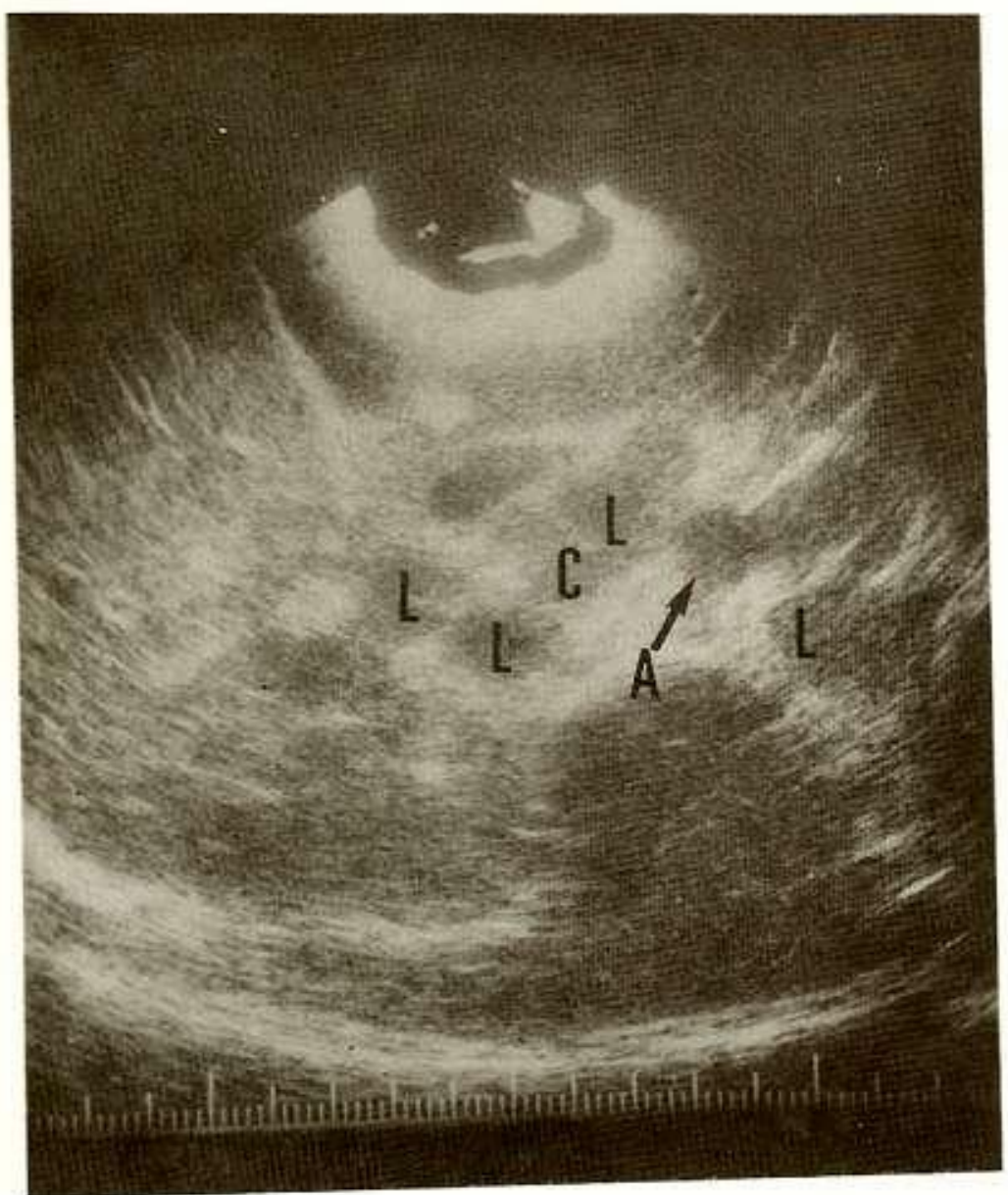

Fig. 1 - Ultrassonografia realiza. da em 27/05/82 mostrando a presença de linfonodos (L) em hilo hopático e regiabo peri-aórtica (A)

\section{CONCLUSÃO}

Embora sejam relativamente frequientes na paracoccidioidomicose, sindromes compressivas de estruturas intratorácicas e principalmente intrabdominais, o presente caso constitui-se no primeiro relato de compressão da veia cava inferior com repercussōes clínicas e hemodinâmicas.

Chamamos a atenção para a possibilidade desse evento em portadores de forma linfo-glandular intrabdominal da paracoccidioidomicose. 
CARVALHO, 5. A de; CERRt. G. G.; SHTROMA. M, SHTCANATYYSUDA, M. A. BARONE, A. A. AR AMATO NETO, V

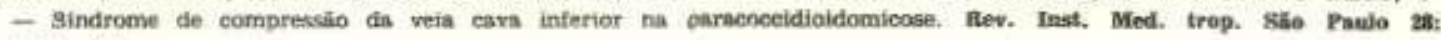
51-64. 1986 .

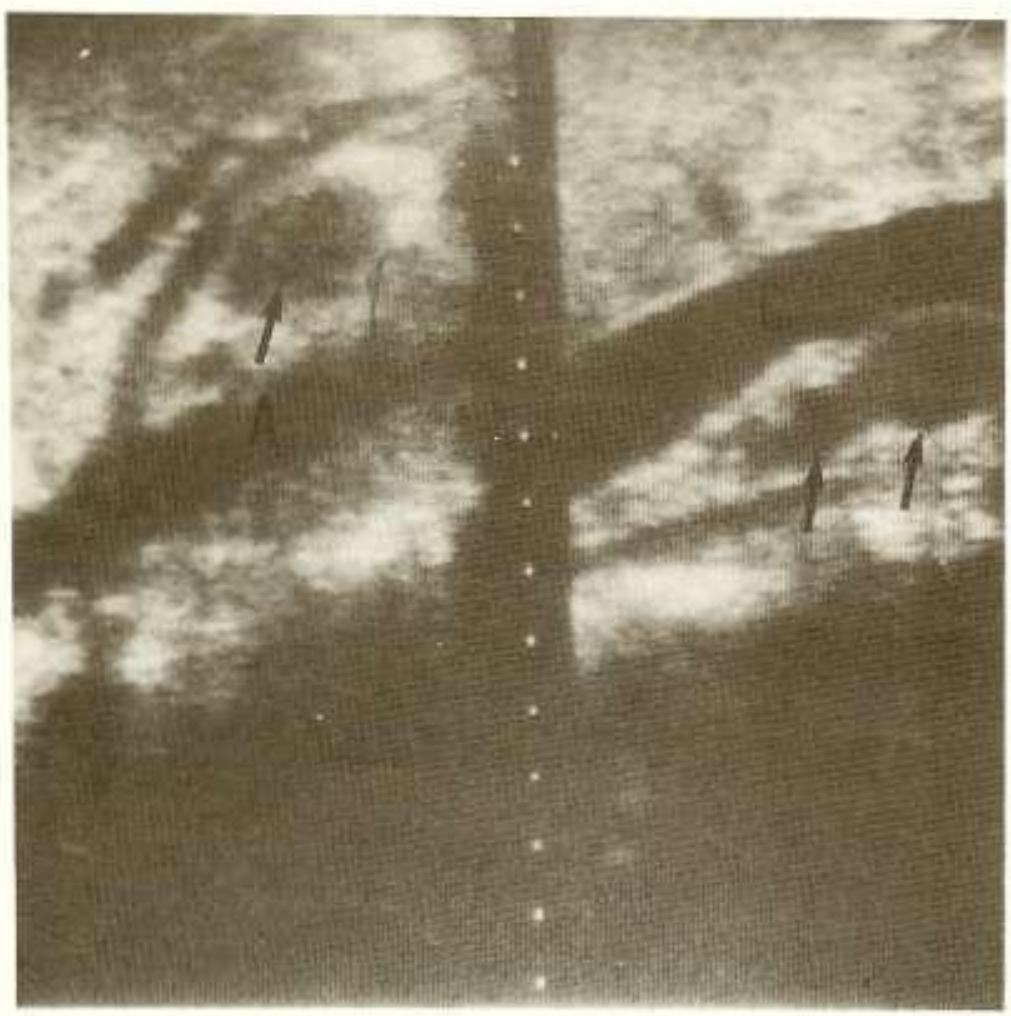

Fig. 2 - Uitrassonogratia realliada em 14/10/82 evidenetando a vela cava interior com o calibre reduzido (C), com imagens bipoe. cogenicas muittiplas em topogrnafia peri-cava e regifo adrtica (A)

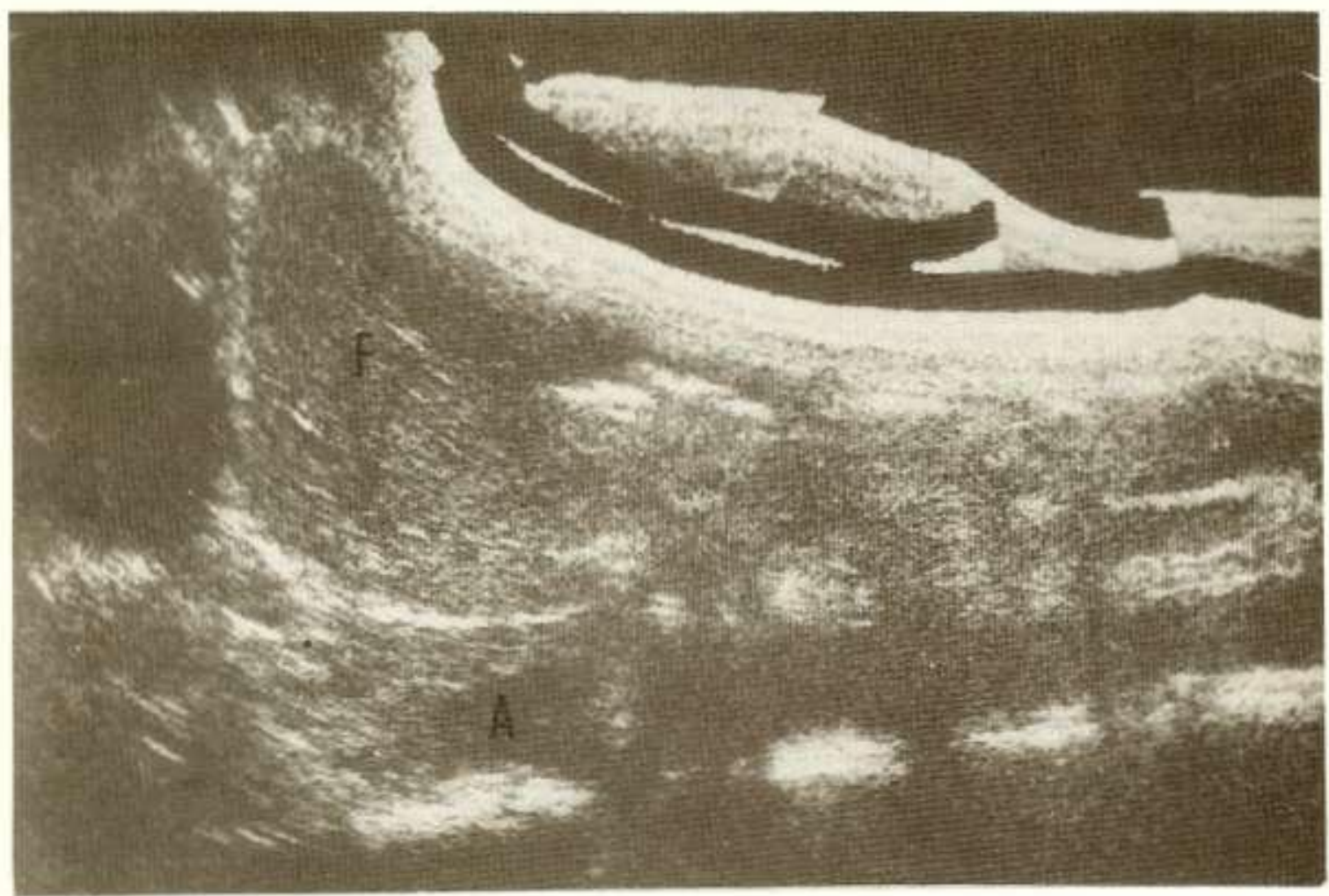

Fie. 3 - Uitrassonografia realiada em 13/12/82, nito evidenciando tinfosdenopatia peri-sortica (A) 
CARVALHO, S. A. de; CERRI, G. G.; SHIROMA, M.; SHIKANAI-YASUDA, M. A.; BARONE, A. A. \& AMATO NETO, Y. - Síndrome de compressāo da veia cava inferior na paracoccidioidomicose. Rev. Inst. Med. trop. Săo Pauto 28: $61.64,1986$.

\section{SUMMARY}

\section{Vena cavae compression syndrome in paracoccidioidomycosis}

The Authors report the first description of an inferior vena cavae compression syndrome due to paracoccidioidomycosis. The clinical course of the disease, laboratory and ultrasonographic findings are summarized, providing evidence to the diagnosis of inferior vena cavae compression.

\section{AGRADECIMENTOS}

Ao Dr. Aluisio Augusto C. Segurado, pela ajuda na realização do presente trabalho.

\section{REFERENGIAS BIBLIOGRAFICAS}

1. ANDRADE, D. R.; HUTZLER, R. U.; CARVALHO, S. A.; ROSENTHAL, C.; CARVALHO, M. A. B. \& FERREIRA, J. M. - Hipoproteinemia em pacientes com paracoccidiofdomicose do tubo digestivo e sistema linfátıco abdominal. Rev. Hosp. Clín. Fac. Med. S. Paulo 31: 174-179, 1976.

2. BARANSKI, M. C.; GOMES, N. R.; MOTA, C. C. S.: CUNHA, C. A. M.; ALENCAR, J. V.; BRENNER, S.; LEAL. W. M. \& BUFFARA, M. - Ictericia obstrutiva na blastomicose sul-americana, apresentaçâo de 2 casos. An. Fac. Med. Paraná 8: 25-39, 1965.

3. BARONE, A. A.; SHIROMA, M. \& AMATO NETO, v. - Paracoccidioidomicose óssea generalizada: relato de um caso. In: Congresso da Sociedade Brasileira de Medicina Tropical XV, Campinas (SP), 1979.

4. BOULOS, M. I. C.; SHIROMA, M.; DUARTE, M. I. S.; SILVEIRA, M. I. \& AMATO NETO, v. - Endocardite paracoccidioidomicótica. In: Congresso da Sociedade Brasileira de Medicina Tropical XVI, Natal (RN), 1980.

5. CESAR, R. C.; CARINI, A.; LAUAND, F. \& LIA, N. - Abdomen agudo de etiologia blastomicótica. Hospitsl (Rio de J.) 61: 625.637, 1962.

6. COSTA, I. A.; BRANDAO, H.; ARTigos, G. V. \& BETTEGA, J. L. - Tratamento cirúrgico de estenose blestomicótica da traquéia. An, parana. Tuberc. 3: 103-109, 1958.
7. DEL NEGRO, G.; LACAZ, C. S. \& FIORIILO, A M. - Paracoccidioidomicose. Săo Paulo, Sarvier-Edusp. 1982

8. FARAGE FILHO, M.; BRAGA, M. R. \& KUHN, M. L. S. - Granuloma blastomicótico na medula cervical. Registro de um caso. Arq. Neuro-psiquiat. (S. Paulo). 35: 151-155, 1977.

9. FAVA NETTO, C. \& DEL NEGRO, G. - Lacalização testiculo-epididimária de blastomicose sul-americana. Rev. Ass. méd. bras. 1: 210-213, 1954.

10. FONSECA, L. C. \& MIGNONE, C. - Paracoccidioidomicose do intestino delgado. Aspectos anátomo-clínicos e radiológicos de 125 casos. Rev. Hosp. Glín. Fac. Med. S. Paulo 31: 199-207, 1976.

11. GONÇALVES, A. J. R.; BARBOSA, L. S. G.; COSTA, I. C.; MORAES, J. C. O. \& GUIMARAES NETO; F. D - Aneurisma blastomicótico da aorta abdominal. Relato de um caso e revisāo da literatura. Rev. méd. Hosp. Serv. Est. 29: 167-172, 1977.

12. MARENGD, R.; CALDAS, E. A. \& RAFFO, J. M. Granuloma paracoccidioiđes com localizaçăo pancreátíca. Sem. méd. (B. Aires) 41: 975-979, 1934.

13. MILAZZO, L. A.; DUARTE, C. M.; AFONSO, C. C. ALBUQUER, J. W.; SILVA, S. T. D; FLORES, A. P. \& BARBOSA, E. T. - Blastomicose sul-americana ou doença de Lutz. Comprometimento cardfaco. Arq. bras. Cardiol. 22 (supt. 1): 70, 1970.

14. MLLINGTON, F. R.; MELLO, M. V.; MILLINGTON, M. A.; FADIGA, E. M.; TAVARES, H. R.; LeITE, E. V.: MEDEIROS, R.; GONÇALVES, A. L. \& LIMA J. M. M. - Manifestaçōes menos frequientes na paracoccidioidomicose. In: Congresso da Sociedade Brasileira de Medicina Tropical, XV. Campinas (SP), 1979.

15. PILHEU, J. A.; YERGA, M.; NEGRONI, R. \& COHEN, J. - Estenose traqueal por Paracoccldioides brasiliensis. Tórax 14: 184-186, 1965.

16. ROCHA, A.; PORTILHO, D. V.; ALMEIDA, H. O; LOPES, E. R. \& SANTOS, S. P. - Abdomen agudo por colecistite blastomicótica. Rev. Goiana Med. 26 (1-2): 63-69, 1980 .

Recebido para publicaçăo em 10/7/1985. 